\title{
FINITE ELEMENT ANALYSIS FOR THE PROGRESSIVE FAILURE OF FRP-REINFORCED STEEL COMPONENT UNDER LOW-VELOCITY IMPACT
}

\author{
Qi-Jian Wu ${ }^{1,2}$, Xu-Dong Zhi ${ }^{1,2,{ }^{*}}$ and Meng-Hui Guo ${ }^{1,2}$ \\ ${ }^{1}$ Key Lab of Structures Dynamic Behavior and Control of the Ministry of Education, Harbin Institute of Technology, Harbin, Chin a \\ ${ }^{2}$ Key Lab of Smart Prevention and Mitigation of Civil Engineering Disasters of the Ministry of Industry and Information Technology, Harbin Institute of Technology, Harbin, China \\ *(Corresponding author: E-mail: zhixudong@hit.edu.cn)
}

\section{A B S T R A C T}

This study proposed a finite element simulation method to analyze the progressive failure of fiber-reinforced polymer (FRP) reinforced steel component under low-velocity impact. In this method, the Johnson-Cook model and fracture criterion were used to consider the strain rate effect of steel; additionally, a VUMAT subroutine was proposed to discuss the 6 initial failure modes (fiber tension/compression failure, matrix tension/compression failure and in-layer tension/compression delamination failure) and damage evolution of FRP. In order to verify the simulation results, a series of axial low-velocity impact tests on GFRP-reinforced circular steel tube were performed, the comparative study confirmed that the simulations were in good agreement with the test results. Besides, the advantages of proposed VUMAT subroutine were made obviously by comparing to the Puck criterion, the Hashin criterion, and the Chang-Chang criterion.
ART I CLE H IS T O RY

$\begin{array}{ll}\text { Received: } & \text { 18 September } 2018 \\ \text { Revised: } & \text { 27 February } 2019 \\ \text { Accepted: } & 6 \text { March } 2019\end{array}$

\section{K E Y W O R D S}

FRP-reinforced steel component Low-velocity impact;

Simulation;

Progress failure;

Damage mechanics

\section{Introduction}

Nowadays, steel components are used widely in important structures due to their advantages of easy construction and high bearing capacity. During the service period of these structures, low-velocity impacts are possible loads and can bring a great loss to the society, such as the collisions by cars or airplanes. Thus, improving the impact resistance of steel component is a necessary way to reduce the collapsed risk of these structures.

Fiber reinforced polymer (FRP) served as energy dissipation member have already been widely applied to the aerospace and automotive manufacturing [1, 2]. Reinforcing the steel component by FRP can harness the advantages of each material and increase both the bearing capacity and energy dissipation capacity $[3,4]$. Besides, the wrapped FRP can also protect the inner steel component from corrosion. Hence, there is a good application prospect of this kind of components in architectural structure.

Because of the advantages of powerful function and saving resources, the finite element analysis has already been extensively applied to the engineering field. The key consideration to simulate FRP-reinforced steel component (FSC) is selecting appropriate constitutive models, including stress-strain relationships, failure criteria, and damage evolution laws. The J-C constitutive model [5, 6] are widely used in metal impact simulation [7-9], since they can consider the metal plastic deformation, the strain rate strengthening effect, and the temperature softening effect. The Hashin criterion [10], the Puck criterion [11], and the Chang-Chang criterion [12] are the most commonly used failure criteria for FRP, nowadays. Many researchers [13-18] had studied the low-velocity impact problems of FRP component based on these three criteria. However, these studies always ignored the in-layer delamination of FRP which is an important failure mode when the thickness of unidirectional FRP lamina is large.

In the present study, a VUMAT subroutine to discuss the 6 initial failure modes (fiber tension/compression failure, matrix tension/compression failure and in-layer tension /compression delamination failure) and damage evolution of FRP was proposed. Besides, based on the VUMAT subroutine and J-C constitutive model, a method to simulate FSC under low-velocity impact was introduced.

\section{Constitutive models for FSC}

\subsection{Stress-strain relationships for steel and FRP}

The mechanical behavior of material is expressed by its stress-strain relationship. Normally, the 3D stress-strain relationship for material is shown in Eq. 1. Where $\sigma_{i}$ is the stress component; $C_{i j}$ is the stiffness coefficient; $\varepsilon_{j}$ is the strain component $(i, j=1,2, \ldots, 6)$.

Steel is an isotropic material so that each point in it has the same elastic properties in any direction. The relationship between the stiffness coefficients of steel is expressed in Eq. 2. $\left(\begin{array}{c}\sigma_{1} \\ \sigma_{2} \\ \vdots \\ \sigma_{6}\end{array}\right)=\left[\begin{array}{cccc}C_{11} & C_{12} & \cdots & C_{16} \\ C_{21} & C_{22} & \cdots & C_{26} \\ \vdots & \vdots & & \vdots \\ C_{61} & C_{62} & \cdots & C_{66}\end{array}\right] \cdot\left(\begin{array}{c}\varepsilon_{1} \\ \varepsilon_{2} \\ \vdots \\ \varepsilon_{6}\end{array}\right)$

$\left\{\begin{array}{l}C_{11}=C_{22}=C_{33} \\ C_{12}=C_{13}=C_{23} \\ C_{44}=C_{55}=C_{66}=1 / 2\left(C_{11}-C_{12}\right)\end{array}\right.$

Engineering parameters can be obtained directly through the material tests. Therefore, these parameters including elasticity modulus $(E)$, Poisson's ratio $(v)$, and shear modulus $(G)$ are typically used to express the stiffness coefficients of steel, as shown in Eq. 3. In this equation, $S=1-2 v^{3}-3 v^{2}$.

$\left(\begin{array}{c}\sigma_{1} \\ \sigma_{2} \\ \sigma_{3} \\ \sigma_{4} \\ \sigma_{5} \\ \sigma_{6}\end{array}\right)=\left[\begin{array}{cccccc}E\left(1-v^{2}\right) / S & E\left(v^{2}+v\right) / S & E\left(v^{2}+v\right) / S & 0 & 0 & 0 \\ E\left(v^{2}+v\right) / S & E\left(1-v^{2}\right) / S & E\left(v^{2}+v\right) / S & 0 & 0 & 0 \\ E\left(v^{2}+v\right) / S & E\left(v^{2}+v\right) / S & E\left(1-v^{2}\right) / S & 0 & 0 & 0 \\ 0 & 0 & 0 & G & 0 & 0 \\ 0 & 0 & 0 & 0 & G & 0 \\ 0 & 0 & 0 & 0 & 0 & G\end{array}\right] \cdot\left(\begin{array}{c}\varepsilon_{1} \\ \varepsilon_{2} \\ \varepsilon_{3} \\ \varepsilon_{4} \\ \varepsilon_{5} \\ \varepsilon_{6}\end{array}\right)$

$\left(\begin{array}{c}\sigma_{1} \\ \sigma_{2} \\ \sigma_{3} \\ \sigma_{4} \\ \sigma_{5} \\ \sigma_{6}\end{array}\right)=\left[\begin{array}{cccccc}\frac{E_{1}\left(1-v_{23} v_{32}\right)}{S} & \frac{E_{2}\left(v_{21}+v_{23} v_{31}\right)}{S} & \frac{E_{3}\left(v_{31}+v_{21} v_{32}\right)}{S} & 0 & 0 & 0 \\ \frac{E_{2}\left(v_{21}+v_{23} v_{31}\right)}{S} & \frac{E_{2}\left(1-v_{13} v_{31}\right)}{S} & \frac{E_{3}\left(v_{32}+v_{12} v_{31}\right)}{S} & 0 & 0 & 0 \\ \frac{E_{3}\left(v_{31}+v_{21} v_{32}\right)}{S} & \frac{E_{3}\left(v_{32}+v_{12} v_{31}\right)}{S} & \frac{E_{3}\left(1-v_{12} v_{21}\right)}{S} & 0 & 0 & 0 \\ 0 & 0 & 0 & G_{23} & 0 & 0 \\ 0 & 0 & 0 & 0 & G_{31} & 0 \\ 0 & 0 & 0 & 0 & 0 & G_{12}\end{array}\right] \cdot\left(\begin{array}{c}\varepsilon_{1} \\ \varepsilon_{2} \\ \varepsilon_{3} \\ \varepsilon_{4} \\ \varepsilon_{5} \\ \varepsilon_{6}\end{array}\right)$

(4)

FRP is an orthotropic material with two orthorhombic elastic symmetry planes, so that its material stiffness matrix has nine independent coefficients $\left(C_{11}, C_{22}, C_{33}, C_{44}, C_{55}, C_{66}, C_{12}=C_{21}, C_{13}=C_{31}, C_{23}=C_{32}\right)$. Similarly, the stiffness coefficients of FRP are usually represented by the engineering parameters, 
including elasticity modulus $\left(E_{i}\right)$, Poisson's ratio $\left(v_{i j}\right)$, and shear modulus $\left(G_{i j}\right.$, where $i, j=1,2,3$, but $i \neq j$ ), as shown in Eq. 4. In this equation, $S=1-v_{12} v_{21}-v_{23} v_{32}-v_{13} v_{31}-2 v_{12} v_{23} v_{31}$.

\subsection{The constitutive model and fracture criterion for steel}

Steel is a strain rate sensitive material so that the strain rate effect can't be ignored during an impact process. The J-C model [5] can consider the metal plastic deformation, the strain rate strengthening effect, and the temperature softening effect; therefore it was chosen to simulate steel, as shown in Eq. 5. In this equation, $A, B, n, C$, and $m$ are the material correlation constants; $\varepsilon_{e q}$ is the equivalent plastic strain; $\dot{\varepsilon}^{*}$ is the dimensionless quivalent plastic strain, where $\dot{\varepsilon}_{0}$ is the reference strain rate; $T^{*}$ is the dimensionless temperature.

$\sigma=\left(A+B \varepsilon_{e q}{ }^{n}\right)\left(1+C \ln \dot{\varepsilon}^{*}\right)\left(1-T^{* m}\right)$

Metal materials fracture because of the formation and expansion of internal microcracks. The development of these microcracks is significantly correlated with the stress state (stress triaxiality), temperature and strain rate. The J-C fracture criterion [6] considered these factors (Eq. 6), which contributes to its widespread use. In Eq. $6, \varepsilon_{f}$ is the failure strain; $d_{1}-d_{5}$ are the material correlation constants; $\sigma^{*}$ is the stress triaxiality and can be calculated by $\sigma^{*}=\sigma_{m} / \sigma_{e q}$, where $\sigma_{e q}$ is the equivalent stress, $\sigma_{m}$ is the hydrostatic pressure.

$$
\varepsilon_{f}=\left[d_{1}+d_{2} \exp \left(d_{3} \sigma^{*}\right)\right]\left(1+d_{4} \ln \dot{\varepsilon}^{*}\right)\left(1+d_{5} T^{*}\right)
$$

Besides, the J-C fracture criterion decouples the damage and the strength. The damage evolution law is represented as Eq. 7, where, $\Delta \varepsilon_{e q}$ is the equivalent plastic strain increment; $D$ is the damage coefficient (the initial value of $D$ is 0 , and when metal fractured the value of $D$ is 1 ). Moreover, the damaged stress component matrix $\boldsymbol{\sigma}^{\mathbf{d}}$ can be represented by Eq. 8 .

$$
D=\sum \frac{\Delta \varepsilon_{e q}}{\varepsilon_{f}}
$$

$$
\boldsymbol{\sigma}^{\mathbf{d}}=\left(\begin{array}{c}
\sigma_{1}^{d} \\
\sigma_{2}^{d} \\
\sigma_{3}^{d} \\
\sigma_{4}^{d} \\
\sigma_{5}^{d} \\
\sigma_{6}^{d}
\end{array}\right)=\left[\begin{array}{cccccc}
1-D & 0 & 0 & 0 & 0 & 0 \\
0 & 1-D & 0 & 0 & 0 & 0 \\
0 & 0 & 1-D & 0 & 0 & 0 \\
0 & 0 & 0 & 1-D & 0 & 0 \\
0 & 0 & 0 & 0 & 1-D & 0 \\
0 & 0 & 0 & 0 & 0 & 1-D
\end{array}\right] \cdot\left(\begin{array}{c}
\sigma_{1} \\
\sigma_{2} \\
\sigma_{3} \\
\sigma_{4} \\
\sigma_{5} \\
\sigma_{6}
\end{array}\right)
$$

\subsection{Failure criteria and damage evolution laws for unidirectional laminate}

Currently, FRP failures are generally considered to be fiber failures, matrix failures, and interlayer delamination failures [13-18]. However, during actual deformation and destruction the in-layer delamination is also a possible failure mode. Thus, there are seven possible failure modes for FRP laminates, including fiber tension/compression failure, matrix tension/compression failure, in-layer stretch/compression delamination failure, and interlayer failure, as shown in Fig. 1. As the basic element of laminated shell, the unidirectional FRP layer should consider the first 6 kinds of failures. A short description of these failure modes and damage evolution laws is included below.

\subsubsection{Fiber tension and compression}

Previous studies $[15,19]$ have shown that the strain was more continuous than the stress during the evolution of damage. Therefore, the Hashin criterion for fiber tension and compression are expressed in the form of strain, as shown in Eq. 9. In this equation, $\varepsilon_{1}^{i t}$ and $\varepsilon_{1}^{i c}$ are the fiber initial failure strains and can be calculated by $\varepsilon_{1}^{i t}=X_{t} / \varepsilon_{1}$ and $\varepsilon_{1}^{i c}=X_{c} / \varepsilon_{1}$, where $X_{t}$ and $X_{c}$ are respectively the fiber tensile and the compressive strength.

$F_{1}^{t}=\left(\frac{\varepsilon_{1}}{\varepsilon_{1}^{i t}}\right)^{2} \geq 1, F_{1}^{c}=\left(\frac{\varepsilon_{1}}{\varepsilon_{1}^{i c}}\right)^{2} \geq 1$

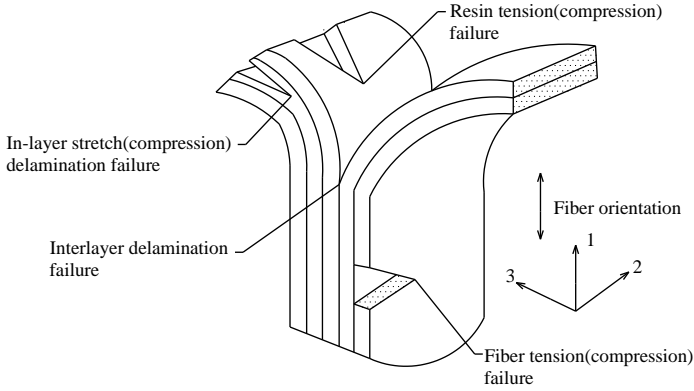

Fig. 1 The possible failure modes of a FRP shell

The damage coefficients for fiber tension and compression are shown in Eq. 10. When denote the finial failure strain $\left(\varepsilon_{1}^{f t}\right.$ and $\left.\varepsilon_{1}^{f c}\right)$, the element characteristic length $l$ is introduced to eliminate the mesh sensitivity problem [15-16], the expression is given by Eq. 11, where $\Gamma_{1}^{t}$ and $\Gamma_{1}^{c}$ are the fiber fracture energy.

$D_{1}^{t}=\frac{\varepsilon_{1}^{f t}}{\varepsilon_{1}^{f t}-\varepsilon_{1}^{i t}}\left(1-\frac{\varepsilon_{1}^{i t}}{\varepsilon_{1}}\right), D_{1}^{c}=\frac{\varepsilon_{1}^{f c}}{\varepsilon_{1}^{f c}-\varepsilon_{1}^{i c}}\left(1-\frac{\varepsilon_{1}^{i c}}{\varepsilon_{1}}\right)$

$\varepsilon_{1}^{f t}=\frac{2 \Gamma_{1}^{t}}{X_{t} l}, \varepsilon_{1}^{f c}=\frac{2 \Gamma_{1}^{c}}{X_{c} l}$

\subsubsection{Matrix tension and compression}

Similar to the fiber tension, the failure criterion and damage coefficient for matrix tension are shown in Eq. 12 and Eq. 13, respectively. In these two equations, $\varepsilon_{2}^{i t}$ and $\varepsilon_{2}^{f t}$ are the matrix initial and final failure strains, respectively; $Y_{t}$ is the matrix tensile strength; $\Gamma_{2}^{t}$ is the matrix tensile fracture energy.

$F_{2}^{t}=\left(\frac{\varepsilon_{2}}{\varepsilon_{2}^{i t}}\right)^{2} \geq 1$

$D_{2}^{t}=\frac{\varepsilon_{2}^{f t}}{\varepsilon_{2}^{f t}-\varepsilon_{2}^{i t}}\left(1-\frac{\varepsilon_{2}^{i t}}{\varepsilon_{2}}\right), \varepsilon_{2}^{i t}=Y_{t} / \varepsilon_{2}, \varepsilon_{2}^{f t}=\frac{2 \Gamma_{2}^{t}}{Y_{t} l}$

Puck et al. [12] introduced a failure criterion that could consider the fracture plane due to matrix compression. Many literatures [14-17] have proved that the Puck criterion have more advantages than the Hashin criterion and the Chang-Chang criterion in discussing the matrix compression. Thus, this criterion was chosen to simulate the matrix compression; moreover, the Puck criterion was also expressed in the form of strain, as shown in Eq. 14. In this equation, $\theta$ is the angle between the fracture plane and the direction of thickness (Puck recommended the value of this angle could be 53 $3^{\circ} ; \varepsilon_{n}(\theta)$, $\gamma_{n l}(\theta)$ and $\gamma_{n t}(\theta)$ are the strain components on the fracture plane, as shown in Fig. 2; $\phi$ is the material friction angle; $E_{23}^{A}$ is the transverse shear strain on the fracture plane; $S_{12}$ is the in-plane shear strength; $G_{12}$ is the in-plane shear modulus.

$$
\left\{\begin{array}{l}
F_{2}^{c}=\left(\frac{\gamma_{n t}(\theta)}{E_{23}^{A}+\mu_{n t} \varepsilon_{n}(\theta)}\right)^{2}+\left(\frac{\gamma_{n l}(\theta)}{S_{12} / G_{12}+\mu_{n l} \varepsilon_{n}(\theta)}\right)^{2} \\
\varepsilon_{n}(\theta)=\varepsilon_{2} \cos ^{2} \theta+\varepsilon_{3} \sin ^{2} \theta+2 \varepsilon_{5} \sin \theta \cos \theta \\
\gamma_{n t}(\theta)=\left(\varepsilon_{3}-\varepsilon_{2}\right) \sin \theta \cos \theta+\varepsilon_{5}\left(\cos ^{2} \theta-\sin ^{2} \theta\right) \\
\gamma_{n l}(\theta)=\varepsilon_{6} \sin \theta+\varepsilon_{4} \cos \theta \\
\mu_{n t}=\tan \left(2 \theta-90^{\circ}\right), \mu_{n l}=\frac{\mu_{n t} S_{12}}{E_{23}^{A} G_{12}} \\
E_{23}^{A}=\frac{Y_{c}}{2 E_{2}^{c}}\left(\frac{1-\sin \phi}{\cos \phi}\right), \phi=2 \theta-90^{\circ}
\end{array}\right.
$$

The damage coefficient of matrix compression can be calculated by Eq 15, where $\gamma_{r}^{i}$ and $\gamma_{r}^{f}$ are the initial and final combined strains, respectively; $\tau_{n t}^{i}(\theta)$ and $\tau_{n l}^{i}(\theta)$ are the shear stresses on the fracture plane at a time of $F_{2}^{c}=1 ; \quad \Gamma_{2}^{c}$ is the matrix compressive fracture energy. 


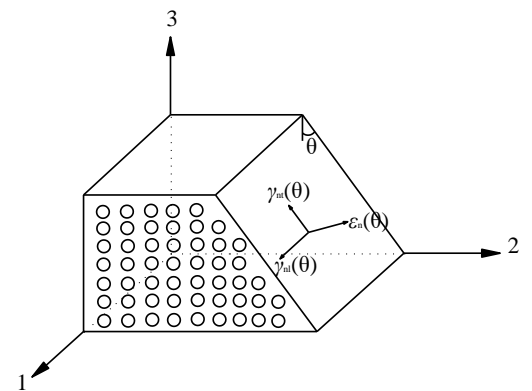

Fig. 2 The fracture plane of matrix under compression

$$
\left\{\begin{array}{l}
D_{2}^{c}=\frac{\gamma_{r}^{f}}{\gamma_{r}^{f}-\gamma_{r}^{i}}\left(1-\frac{\gamma_{r}^{i}}{\gamma_{r}}\right) \\
\gamma_{n t}(\theta)=\left(\varepsilon_{3}-\varepsilon_{2}\right) \sin \theta \cos \theta+\varepsilon_{5}\left(\cos ^{2} \theta-\sin ^{2} \theta\right) \\
\gamma_{n l}(\theta)=\varepsilon_{6} \sin \theta+\varepsilon_{4} \cos \theta \\
\gamma_{r}=\sqrt{\gamma_{n t}^{2}(\theta)+\gamma_{n l}^{2}(\theta)} \\
\gamma_{r}^{f}=\frac{2 \Gamma_{2}^{c}}{S_{r}^{i} l}, \quad S_{r}^{i}=\sqrt{\left(\tau_{n t}^{i}(\theta)\right)^{2}+\left(\tau_{n l}^{i}(\theta)\right)^{2}}
\end{array}\right.
$$

\subsubsection{In-layer delamination}

In order to consider the in-layer delamination, the present study defined the failure criterion and the damage coefficients for the in-layer delamination as shown in Eq. 16 and Eq. 17. In these equations, $Z_{t}$ and $Z_{c}$ are the tension and compression strength in the thickness direction of unidirectional laminate, respectively; $\varepsilon_{3}^{i t}$ and $\varepsilon_{3}^{f t}$ are the initial and final failure strains, respectively; $\varepsilon_{3}^{i c}$ and $\varepsilon_{3}^{f c}$ are the initial and final failure strains, respectively; $\Gamma_{1}^{t}$ and $\Gamma_{1}^{c}$ are the fracture energy in the thickness direction of unidirectional laminate.

$F_{3}^{t}=\left(\frac{\varepsilon_{3}}{\varepsilon_{3}^{i t}}\right)^{2} \geq 1, F_{3}^{c}=\left(\frac{\varepsilon_{3}}{\varepsilon_{3}^{i c}}\right)^{2} \geq 1$

$$
\left\{\begin{array}{l}
D_{3}^{t}=\frac{\varepsilon_{3}^{f t}}{\varepsilon_{3}^{f t}-\varepsilon_{3}^{i t}}\left(1-\frac{\varepsilon_{3}^{i t}}{\varepsilon_{3}}\right), \varepsilon_{3}^{f t}=\frac{2 \Gamma_{3}^{t}}{Z_{t} l} \\
D_{3}^{c}=\frac{\varepsilon_{3}^{f c}}{\varepsilon_{3}^{f c}-\varepsilon_{3}^{i c}}\left(1-\frac{\varepsilon_{3}^{i c}}{\varepsilon_{3}}\right), \varepsilon_{3}^{f c}=\frac{2 \Gamma_{3}^{c}}{Z_{c} l}
\end{array}\right.
$$

Because the strength of matrix is much lower than fiber, the matrix in a FRP unidirectional layer is broken easier. As a result, the in-layer delamination is always caused by matrix fracture in the thickness direction; and the strength and fracture energy in this direction can be regarded approximately equal to the strength and fracture energy of matrix.

\subsubsection{The stress-strain relationships after initial failure}

Once the initial failure occurred, the FRP unidirectional lamina would begin to damage. The damaged stress component $\sigma^{\mathbf{d}}$ can be written by Eq. 18 , where $D_{i}(i=1,2, \ldots, 6)$ is the component of damage matrix $\mathbf{D}$ and can be defined by the damage coefficients (Eq. 19). In Eq. 19, $S_{m t}$ and $S_{m c}$ are the parameters to control the shear stiffness loss caused by matrix tension and compression. According to the literature [13], the loss control factors are postulated as $S_{m t}=0.9$ and $S_{m c}=0.5$.

\subsection{Interlaminar damage model}

In actual engineering, the FRP components are always consisted of many different unidirectional layers, which mean the interlayer failure should be considered. Especially for the FRP-reinforced steel component, the interlaminar damage is caused by interface cracking (where the interface is the matrix that connects two adjacent layers, and the steel component can be seen as one layer). This failure is always simulated using a bilinear traction-separation relationship (Fig. 3) in commercial FEM software, such as the cohesive behavior in ABAQUS/Explicit [20].
$\boldsymbol{\sigma}^{\mathbf{d}}=\mathbf{D} \cdot \boldsymbol{\sigma} \Rightarrow\left(\begin{array}{c}\sigma_{1}^{d} \\ \sigma_{2}^{d} \\ \sigma_{3}^{d} \\ \sigma_{4}^{d} \\ \sigma_{5}^{d} \\ \sigma_{6}^{d}\end{array}\right)=\left[\begin{array}{cccccc}1-D_{1} & 0 & 0 & 0 & 0 & 0 \\ 0 & 1-D_{2} & 0 & 0 & 0 & 0 \\ 0 & 0 & 1-D_{3} & 0 & 0 & 0 \\ 0 & 0 & 0 & 1-D_{4} & 0 & 0 \\ 0 & 0 & 0 & 0 & 1-D_{5} & 0 \\ 0 & 0 & 0 & 0 & 0 & 1-D_{6}\end{array}\right] \cdot\left(\begin{array}{l}\sigma_{1} \\ \sigma_{2} \\ \sigma_{3} \\ \sigma_{4} \\ \sigma_{5} \\ \sigma_{6}\end{array}\right)(18)$

$\left\{\begin{array}{l}D_{1}=\max \left(D_{1}^{t}, D_{1}^{c}\right) \\ D_{2}=\max \left(D_{2}^{t}, D_{2}^{c}\right) \\ D_{3}=\max \left(D_{3}^{t}, D_{3}^{c}\right) \\ D_{4}=1-\left(1-D_{1}^{t}\right)\left(1-D_{1}^{c}\right)\left(1-S_{m t} D_{2}^{t}\right)\left(1-S_{m c} D_{2}^{c}\right) \\ D_{5}=1-\left(1-S_{m t} D_{2}^{t}\right)\left(1-S_{m c} D_{2}^{c}\right)\left(1-S_{m t} D_{3}^{t}\right)\left(1-S_{m c} D_{3}^{c}\right) \\ D_{6}=1-\left(1-D_{1}^{t}\right)\left(1-D_{1}^{c}\right)\left(1-S_{m t} D_{3}^{t}\right)\left(1-S_{m c} D_{3}^{c}\right)\end{array}\right.$

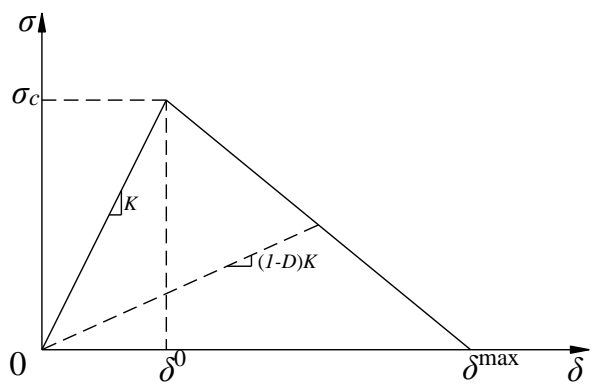

Fig. 3 The bilinear traction-separation relationship

There are three kinds of stresses on the interface (normal stress $S_{n}$, shear stress $S_{t}$, and $\left.S_{l}\right)$, which are defined within Eq. 20. In this equation, $G_{i}(i=n, t, l)$ is the critical strain energy release rate, which is the area covered by the $\sigma-\delta$ curve.

$\left\{\begin{array}{l}\int_{0}^{\delta_{\max }} S_{n} \mathrm{~d} \delta_{n}=G_{n} \\ \int_{0}^{\delta_{t}^{\max }} S_{t} \mathrm{~d} \delta_{t}=G_{t} \\ \int_{0}^{\delta_{t}^{\max }} S_{l} \mathrm{~d} \delta_{l}=G_{l}\end{array}\right.$

The stress-strain relationship on the interface is represented by Eq. 21 Where $K_{i}(i=n n, t t, l l)$ denotes the slope of Fig. 3 and $D^{I}$ is the damage coefficient for interlaminar damage.

$\left(\begin{array}{l}S_{n} \\ S_{t} \\ S_{l}\end{array}\right)=\left[\begin{array}{ccc}\left(1-D^{I}\right) K_{n n} & 0 & 0 \\ 0 & \left(1-D^{I}\right) K_{t t} & 0 \\ 0 & 0 & \left(1-D^{I}\right) K_{l l}\end{array}\right] \cdot\left(\begin{array}{l}\varepsilon_{n} \\ \varepsilon_{t} \\ \varepsilon_{l}\end{array}\right)$

The interlaminar damage process is as follows. When the interface is in elastic state $\left(\delta \leq \delta^{0}\right)$, the value of damage coefficient $D^{I}$ is 0 ; once it begins to damage ( $\delta^{0}<\delta<\delta^{\max }$ ), the damage evolution law is expressed in Eq. 22; finally, the interface totally fractured $\left(\delta \geq \delta^{\max }\right)$, the $D^{I}$ reaches a value of 1

$D^{I}=\frac{\delta_{m}^{f}\left(\delta_{m}^{\max }-\delta_{m}^{0}\right)}{\delta_{m}^{\max }\left(\delta_{m}^{f}-\delta_{m}^{0}\right)}, \quad \delta_{m}=\sqrt{\delta_{n}^{2}+\delta_{t}^{2}+\delta_{l}^{2}}$

In Eq. 22, $\delta_{m}^{f}$ is the mixed-mode displacement, which is defined by the Benzeggagh-Kenane fracture criterion [21] (as shown in Eq. 23, where $\xi$ and $\eta$ are the Benzeggagh-Kenane law parameters). 
$\delta_{m}^{f}= \begin{cases}\frac{2}{K \delta_{m}^{0}}\left[G_{I C}+\left(G_{I C}-G_{I C}\right) \xi^{\eta}\right] & \delta_{n}>0 \\ \sqrt{\left(\delta_{t}^{f}\right)^{2}+\left(\delta_{l}^{f}\right)^{2}} & \delta_{n} \leq 0\end{cases}$

\section{Simulation method}

\subsection{Axial low-velocity impact tests}

A series of impact tests were developed to verify the simulations. The specimen was manufactured through a filament winding process (Fig. 4), and had a GFRP enhancement layer (S2-1200 glass fiber reinforced E-51 epoxy resin) and a circular Q235 steel tube base layer (Fig. 5). A total of 6 conditions were discussed in the tests, as shown in Table 1; moreover, 58-1.5-30-3 was replicated thrice to verify the stability of tests.

The tests were conducted on a drop hammer test system, as shown in Fig. 6. During the test process, the specimen was anchored in a $60 \mathrm{~mm}$ deep steel groove using high strength gypsum; besides, the impact force was obtained by a piezoelectric force transducer; and a magnet-grid linear displacement system was used to measure the displacement of hammer. Additionally, a high-speed camera (Fig. 7) was used to record the axial impact process.

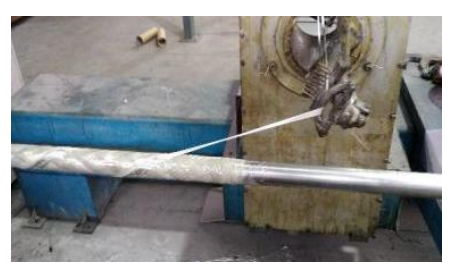

Fig. 4 The filament winding process

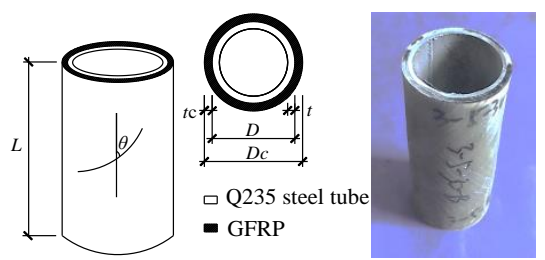

Fig. 5 A detail of the specimens tested

Table 1

Descriptions of the specimens tested

\begin{tabular}{|c|c|c|c|c|c|c|c|}
\hline Label & $D / \mathrm{mm}$ & $\mathrm{t} / \mathrm{mm}$ & {$[\theta]_{n}$} & $D_{c} / \mathrm{mm}$ & $t c / \mathrm{mm}$ & $L / \mathrm{mm}$ & $\mathrm{V} / \mathrm{m} / \mathrm{s}$ \\
\hline $58-1.5-30-3$ & 58.0 & 1.5 & {$[30 /-30]_{3}$} & 62.0 & 2.0 & 100.0 & 3.0 \\
\hline $58-1.5-90-3$ & 58.0 & 1.5 & {$[90]_{9}$} & 62.0 & 2.0 & 100.0 & 3.0 \\
\hline $58-1.5-30-4.5$ & 58.0 & 1.5 & {$[30 /-30]_{3}$} & 62.0 & 2.0 & 100.0 & 4.5 \\
\hline $58-1.5-45-4.5$ & 58.0 & 1.5 & {$[45 /-45]_{3}$} & 62.0 & 2.0 & 100.0 & 4.5 \\
\hline $58-1.5-90-4.5$ & 58.0 & 1.5 & {$[90]_{9}$} & 62.0 & 2.0 & 100.0 & 4.5 \\
\hline
\end{tabular}

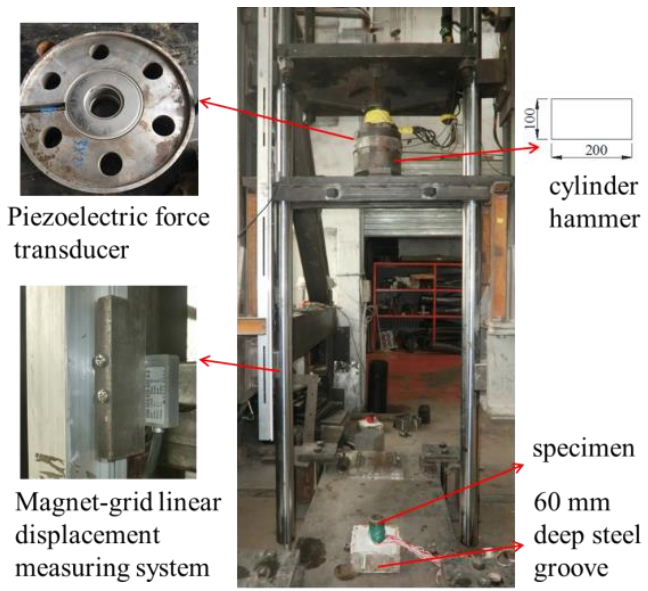

Fig. 6 The drop hammer test system

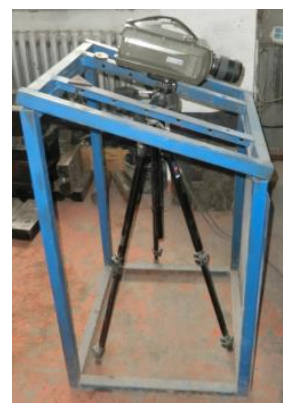

Fig. 7 High-speed camera

The three repeated test results of 58-1.5-30-3 were shown in Fig. 8. The results were compared form 4 aspects: impact load (load-time curve, as shown in Fig. 8 (a)), axial displacement (displacement-time curve, as shown in Fig. 8 (b)), energy dissipation (the cover area of load-displacement curve, as shown in Fig. 8 (c)), and damage modes (as shown in Fig. 8 (d)). In conclusion, the results of the parallel samples were easily reproducible, and hence the test results obtained from the drop hammer test system were credible.

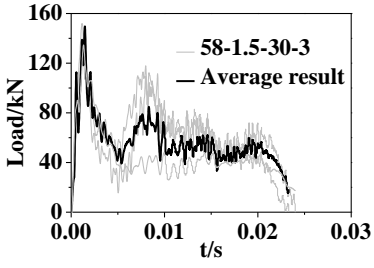

(a) Load-time curves

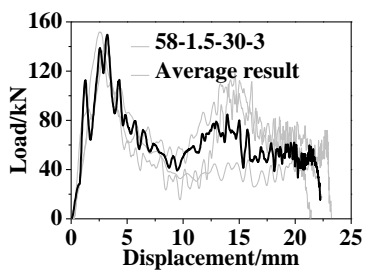

(c) Load-displacement curve

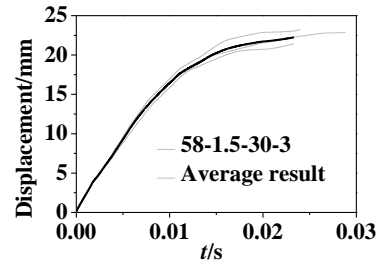

(b) Displacement-time curves

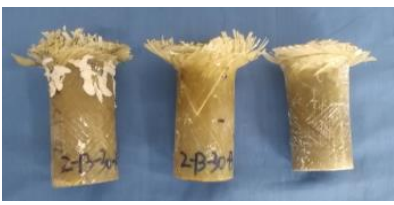

(d) Damage specimens
Fig. 8 Repeated test results of 58-1.5-30-3

\subsection{Finite element model}

ABAQUS 6.11/Explicit was chosen to develop the simulation works Each layer was modeled separately (for both FRP layers and steel layers), such that the damage development in each individual layer could be simulated. In the thickness direction of each layer there was one element, including the C3D8R (8-node linear brick, reduced integration, hourglass control [20]) for FRP plies and C3D8I (8-node linear brick, incompatible modes [20]) for steel ply. The drop hammer was modeled by C3D10M (10-node modified quadratic tetrahedron [20]). The bottom of the specimen was completely clamped to the support, while its top was left free. The impact load was applied to the specimen through the drop hammer's mass and initial velocity. The finite 
element model is shown in Fig. 9.

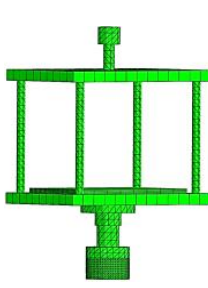

(a) Drop hammer

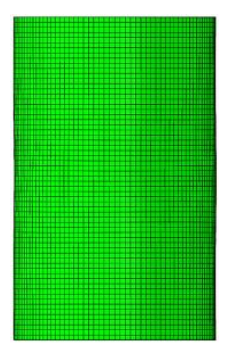

(b) Specimen

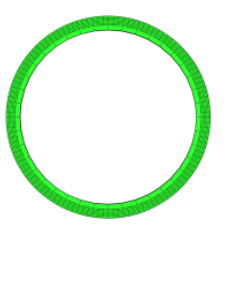

(c) Assembly drawing
Fig. 9 The finite element model

\subsection{Material parameters}

The steel layer was simulated by the J-C constitutive model provided by ABAQUS. Its quasi-static material properties were obtained using uniaxial tensile tests, where the results for these tests are shown in Table 2. And the parameters for $\mathrm{J}-\mathrm{C}$ model were determined according to the previous study [7], as shown in Table 3 .

Table 2

Mechanical properties of the steel tubes [22]

\begin{tabular}{cccccc}
\hline$t / \mathrm{mm}$ & $\sigma_{y} / \mathrm{MPa}$ & $\sigma_{u} / \mathrm{MPa}$ & $\varepsilon_{y} / \mu \varepsilon$ & $\varepsilon_{u} / \mu \varepsilon$ & $E / \mathrm{GPa}$ \\
\hline $1.5 / 2.0$ & 272.28 & 410.64 & 1991 & 240000 & 208 \\
\hline
\end{tabular}

As for FRP a VUMAT subroutine based on the mechanics of composite material was developed. The subroutine can consider the 6 initial failure modes and damage evolutions of FRP unidirectional laminate, its computational algorithm is shown in Fig. 10. Because GFRP is anisotropic, its properties were obtained by tensile tests (longitudinal and transverse), compression tests (longitudinal and transverse), interlayer shear-strength tests, in-plane shear tests, and debonding test. The properties of S2-1200 glass fiber/E-51 epoxy unidirectional laminate are shown in Table 4 [22]. Moreover, the interfaces between steel layer and GFRP layer, or between GFRP layers, were simulated by the cohesive behavior provided by ABAQUS, where these cohesive parameters are shown in Table 5 [22]

Table 3

Parameters of the Johnson-Cook model and fracture criterion [7]

\begin{tabular}{ccccccccccccc}
\hline$A / \mathrm{MPa}$ & $B / \mathrm{MPa}$ & $n$ & $m$ & $T_{m} / \mathrm{K}$ & $T_{r} / \mathrm{K}$ & $D 1$ & $D 2$ & $D 3$ & $D 4$ & $\dot{\varepsilon}_{0} / \mathrm{s}^{-1}$ & \\
\hline 272.28 & 899.7 & 0.94 & 0.1515 & 1795 & 293 & -43.408 & 44.608 & 0.016 & 0.0145 & -0.046 \\
\hline
\end{tabular}

Table 4

Material properties of GFRP unidirectional laminate materials [22]

\begin{tabular}{cc}
\hline Parameters & Value \\
\hline Modulus/GPa & $E_{1}=41.29 ; E_{2}=E_{3}=4.21 ; G_{12}=G_{13}=3.16 ; G_{23}=3.0$ \\
Poisson's ratio & $v_{12}=v_{13}=0.31 ; v_{23}=0.42$ \\
Strength/MPa & $X_{t}=884.5 ; X_{c}=837.17 ; Y_{t}=Z_{t}=37.38 ; Y_{c}=Z_{c}=145 ;$ \\
& $S_{12}=S_{13}=44.765 ; S_{23}=50.88$ \\
Fracture energy/( N/mm) & $\Gamma_{1}^{t}=28.25 ; \Gamma_{1}^{c}=80.1 ; \Gamma_{2}^{t}=\Gamma_{3}^{t}=0.36 ;$ \\
& $\Gamma_{2}^{c}=\Gamma_{3}^{c}=7.24$ \\
\hline
\end{tabular}

Table 5

Cohesive behavior parameters [22]

\begin{tabular}{cc}
\hline Parameters & Value \\
\hline Elastic properties/GPa & $K_{n n}=4210 ; K_{t l}=3160 ; K_{l l}=3160$ \\
Strength/MPa & $T_{n}=37.38 ; T_{t}=44.765 ; T_{l}=44.765$ \\
Fracture toughness/(N/mm) & $G_{n}=0.36 ; G_{t}=1.33 ; G_{l}=1.33$ \\
Mode interaction-BK & $\eta=2.6$ \\
\hline
\end{tabular}

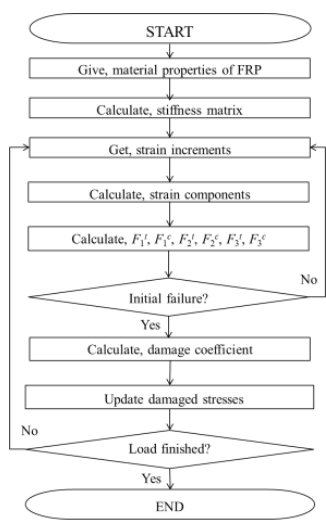

Fig. 10 Computational algorithm of the VUMAT subroutine

The drop hammer was assumed to keep elastic during the impact process. Its material properties were defined as $E=217 \mathrm{GPa}, v=0.3$. The contact between the drop hammer and FSC was simulated using the penalty method; according to Perillo's study [23], an average friction coefficient of $\mu=0.3$ was appropriate.

\subsection{Verification of the simulation method}

The measured results including load-displacement curves and energy displacement curves were compared with simulations, as shown in Fig. 11 Compared to the simulations the load-displacement curves obtained from the tests exhibited larger vibrations, this was because the drop hammer would vibrate during axial impact process. Besides, the specimens in the impact tests inevitably had some initial imperfections; however, these imperfections were ignored in the finite element simulation to simplify the analysis, so that the simulations were a little larger than the test results. Even so, the simulations still have a good agreement with the test results.
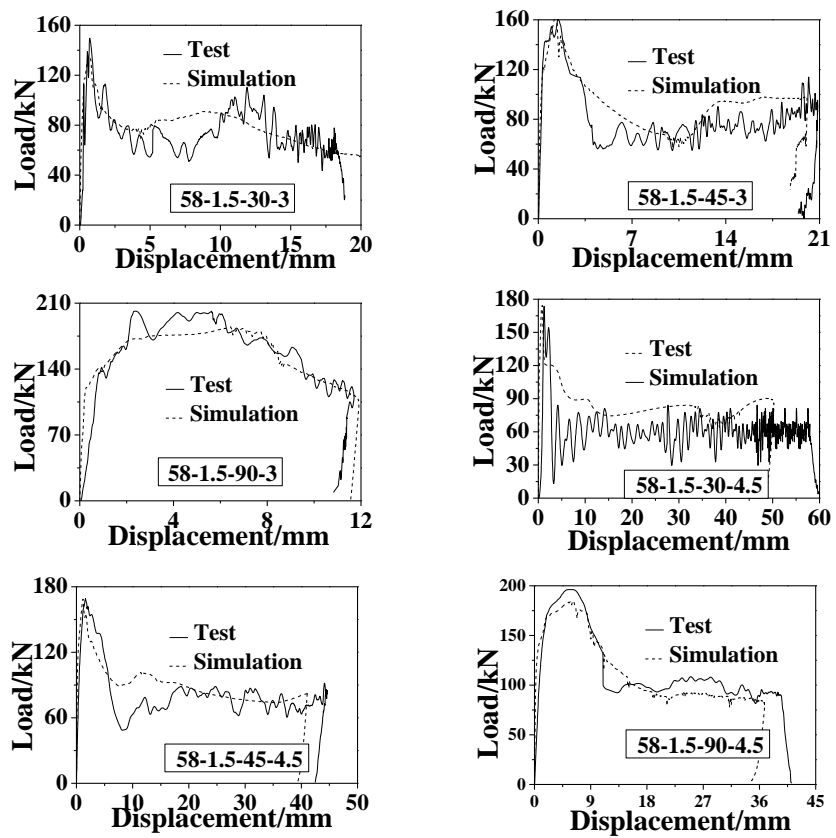

(a) Load-displacement curves 

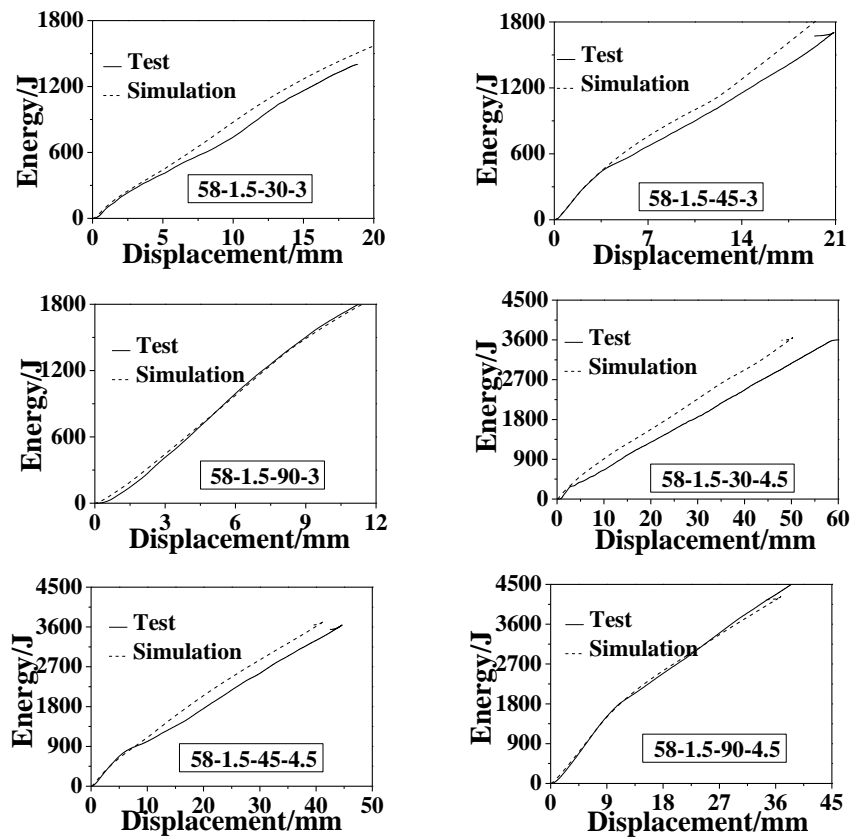

(b) Energy-displacement curve

Fig. 11 Compared between experimental and simulated results

Take the specimens with impact velocity $3 \mathrm{~m} / \mathrm{s}$ for example. Fig. 12 shows a comparison of damage modes between the tests and the simulations. Moreover, Fig. 13 detailedly compares the impact process of 58-1.5-45-4.5. The axial low-velocity impact process can be divided into two stages: elastic stage and energy dissipation stage. During the elastic stage GFRP and steel were in an elastic state, where the sample kept vertical and its midspan expanded along the radial direction $(0-0.001$ seconds for the test, and $0-0.000825$ seconds for the simulation). After this, the specimen entered an energy dissipation stage until the end of the first impact process (0.001-0.0295 s for the test, and 0.000825-0.0292 $\mathrm{s}$ for the simulation). During this stage, the steel tube dissipated energy through plastic deformation, and the FRP dissipated energy through both fiber and resin failure and delamination damage. According to the comparison, the simulated damage modes and impact process matched the tests well.

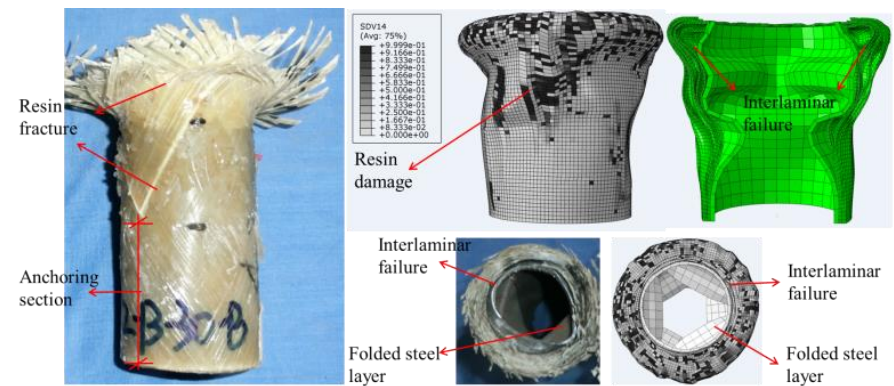

(a) $58-1.5-30-3$

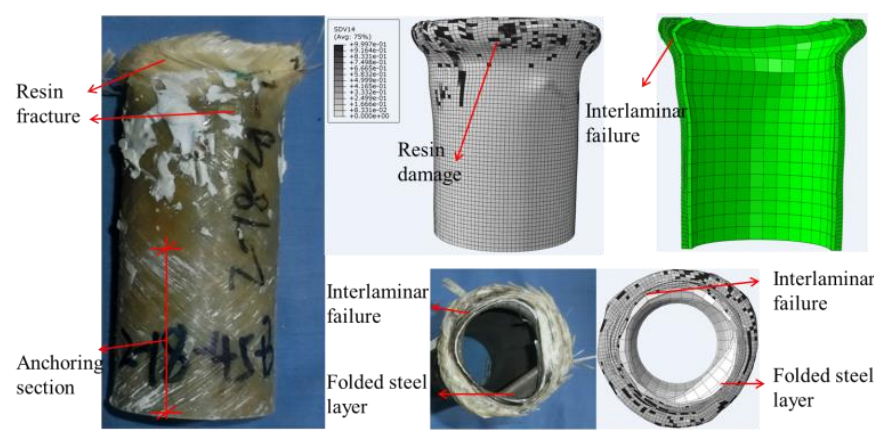

(b) $58-1.5-45-3$
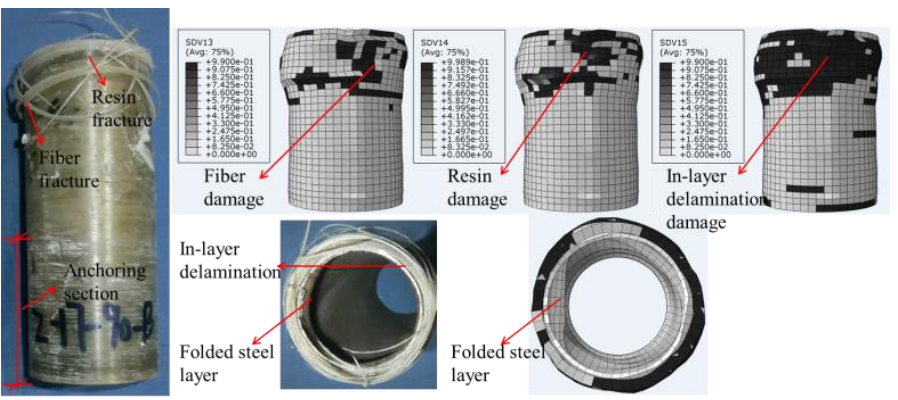

(c) $58-1.5-90-3$

Fig. 12 Comparing damage between predicted and tested samples

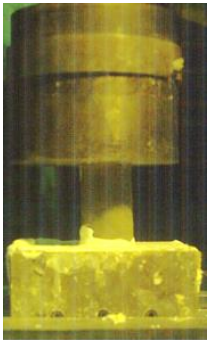

$\mathrm{t}=0 \mathrm{~s}$

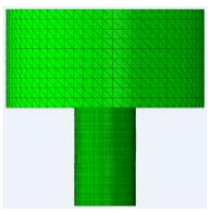

$\mathrm{t}=0 \mathrm{~s}$

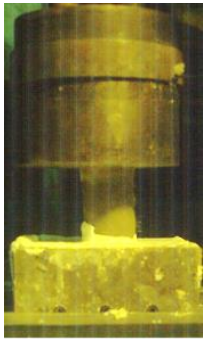

$\mathrm{t}=0.001 \mathrm{~s}$

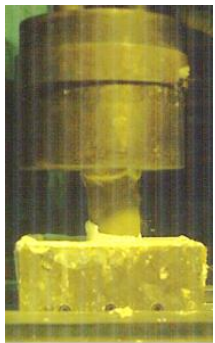

$\mathrm{t}=0.002 \mathrm{~s}$

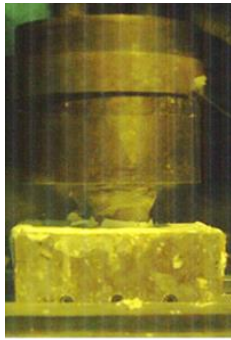

$\mathrm{t}=0.0295 \mathrm{~s}$ (a) Experimental results

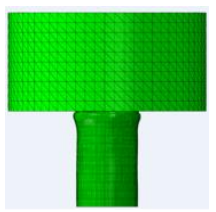

$\mathrm{t}=0.000825 \mathrm{~s}$
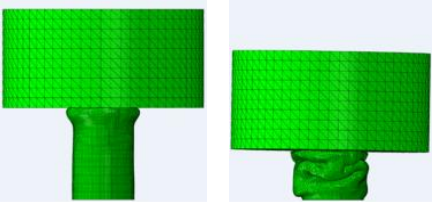

$\mathrm{t}=0.0292 \mathrm{~s}$

(b) Predicted result

Fig. 13 Compared damage process of 58-1.5-45-4.5 between predicted and experimental

\section{Comparison between common failure criteria and VUMAT}
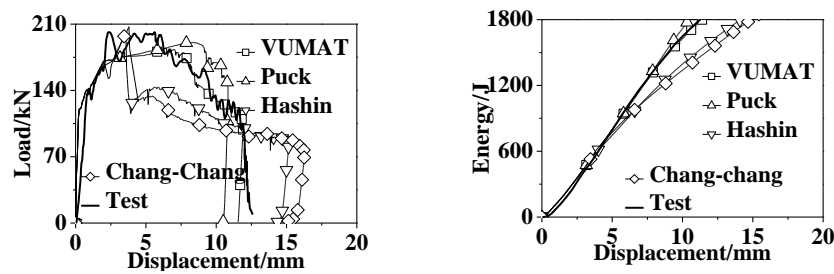

(a) $58-1.5-90-3$
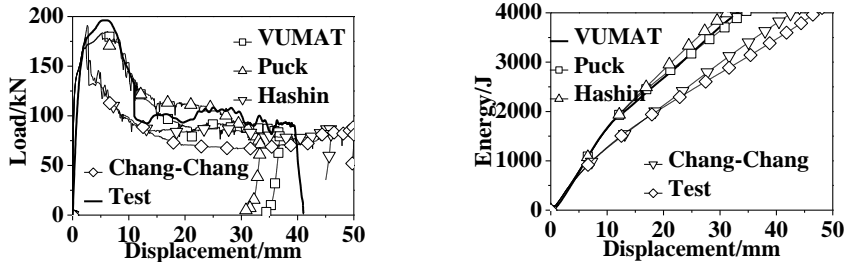

(b) $58-1.8-90-4.5$

Fig. 14 Results of 58-1.5-90-3 and 58-1.8-90-4.5 using different failure criteria

To show the advantages of the proposed VUMAT subroutine, 58-1.5-90-3 and 58-1.5-90-4.5 were simulated using the Puck criterion, the Hashin criterion, the Chang-Chang criterion, and the VUMAT subroutine (where the steel tube used the J-C model). Results of this comparison are shown in Fig. 14. Because of considering the influence of fracture plane, the results from the Puck criterion and the VUMAT subroutine were better than them from the Hashin criterion and the Chang-Chang criterion. To be specific, the curves from the Hashin criterion and the Chang-Chang criterion had a sharper decrease in the load-descending phase, and their shapes are thinner than the experimental results (Fig. 14 (a) and (c)); additionally, the energy-dissipation simulated by these two criteria was distinctly lower than the experimental results (Fig. 14 (b) and (d)). 
There was relatively good agreement among the results for the Puck criterion, the VUMAT subroutine, and the experimental results when the impact energy was $1800.9 \mathrm{~J}$ or $4052.025 \mathrm{~J}$ (Fig. 14); however, since the Puck criterion did not consider the in-layer delamination, its results were a little higher than the results for either the VUMAT subroutine or the experimental results. This tendency was more distinct when the impact energy increased, as shown in Fig. 15 (where data in Fig. 15 were obtained via simulation).

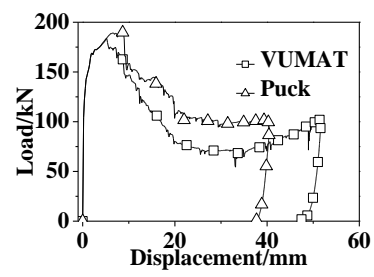

(a) Load-displacement curves

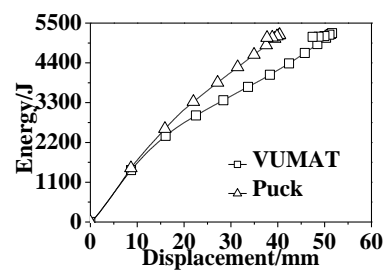

(b) Energy-displacement curves
Fig. 15 Results from samples 58-1.5-90-5.5 using the Puck criterion and the VUMAT
This paper proposed a method to simulate the low-velocity impact process of FRP-reinforced steel component. This method used the $\mathrm{J}-\mathrm{C}$ model to simulate the steel component, and developed a VUMAT subroutine to analyze the initial failures and damage evolution of FRP. Different from the common FRP failure criteria, the proposed subroutine considered not only the fiber tension/compression failure and the matrix tension/compression failure, but the in-layer stretch/compression delamination failure. Moreover, when initial failure occurred, the progressive damage evolution law based on strain was applied in the subroutine.

Axial low-velocity impact tests were developed on GFRP-reinforced circular steel tubes to verify the simulations. This comparative study confirmed that the simulations agreed well with experimental tests not only for load-displacement curves, but also for damage modes. Additionally, a comparison among the Puck criterion, the Hashin criterion, the Chang-Chang criterion and the VUMAT subroutine indicated that the proposed simulation method can simulate the FSC better.

\section{Acknowledgements}

The financial support of Chinese National Natural Science Foundation (Grant No. 51478144 and No. 51778183) is gratefully acknowledged.

\section{Conclusions}

\section{References}

[1] Siromani D., Henderson G., Mikita D., et al. "An experimental study on the effect of failure trigger mechanisms on the energy absorption capability of CFRP tubes under axial compression", Composites: Part A, 64, 25-35, 2014

[2] Song H.W., Wan Z.M., Xie Z.M. and Du X.W., "Axial impact behavior and energy absorption efficiency of composite wrapped metal tubes", International Journal of Impact Engineering, 24, 385-401, 2000.

[3] Bambach, M.R., "Axial capacity and crushing of thin-walled metal, fibre-epoxy and composite metal-fibre tubes", Thin-walled structures, 48, 440-452, 2010.

[4] Harries Kent A., Peck Andrew J. and Abraham Elizabeth J., "Enhancing stability of structural steel sections using FRP”, Thin-walled structures, 47, 1092-1101, 2009.

[5] Johnson G.R. and Cook W.H., "A Constitutive Model and Data for Metals Subjected to Large Strains, High Strain Rates and High Temperatures", Proceedings of the seventh international symposium on ballistics, 541-7, 1983.

[6] Johnson G.R. and Cook W.H., Fracture characteristics of three metals subjected to various strains, strain rates, temperatures and pressures", Eng. Fract. Mech., 21, 31-48, 1985.

[7] Lin L., Fan F. and Zhi X.D., "Dynamic constitutive relation and fracture model of Q235A steel", Appl. Mech. Mater., 274, 463-6, 2013.

[8] Senthil K., Iqbal M.A., Chandel P.S., et al., "Study of the Constitutive Behavior of 7075-T651 Aluminum Alloy", Int. J. Impact Eng., 108, 171-90, 2017.

[9] Wang X. and Shi J., "Validation of Johnson-Cook plasticity and damage model using impact experiment", Int. J. Impact Eng., 60, 67-75, 2013.

[10] Hashin Z., "Failure criterion for unidirectional fiber composite", J. Appl. Mech., 47, 329-34, 1980.

[11] Chang F.K. and Chang K.Y., "A progressive damage model for laminated composites containing stress concentrations", J. Compos. Mater", 21(2), 834-55, 1987.

[12] Puck A. and Schürmann H., "Failure analysis of FRP laminates by means of physically based phenomenological models", Compos. Sci. Technol., 42, 1633-62, 2002.

[13] Lee C.S., Kim J.H., Kim S.K., et al., "Initial and progressive failure analyses for composite laminates using Puck failure criterion and damage-coupled finite element method. Composite Structures", 121, 406-419, 2015.

[14] Singh H., Mahajan P. and Namala K.K., "A Progressive Failure Study of E-glass Epoxy Composite in Case of Low Velocity Impact", Advances in Structural Engineering, 273-300, 2015 .

[15] Liu P.F., Liao B.B., Jia L.Y., et al. "Finite element analysis of dynamic progressive failure of carbon fiber composite laminates under low velocity impact", Composite Structures,149, 408-422, 2016

[16] Liao B.B. and Liu P.F., "Finite element analysis of dynamic progressive failure of plastic composite laminates under low velocity impact", Composite Structures, 159, 567-578, 2017.

[17] Shi Y., Swait T and Soutis C., "Modelling damage evolution in composite laminates subjected to low velocity impact”, Composite Structures, 94, 2902-2913, 2012.

[18] Singh H., Namala K.K. and Mahajan P.A. "Damage evolution study of E-glass/epoxy composite under low velocity impact", Composites: Part B, 76, 235-248, 2015.

[19] Huang C.H. and Lee Y.J., "Experiments and simulation of the static contact crush of composite laminated plates", Composite Structures, 61, 265-70, 2003.

[20] ABAQUS I. ABAQUS 6.11 User's manual, 2011.

[21] Camanho P.P. and Davila C.G., "Mixed-mode decohesion finite elements for the simulation of delamination in composite materials", Tech Rep NASA/TM-2002-211737, 2002.

[22] Zhi X.D., Wu Q.J. and Wang C., "Experimental and numerical study of GFRP-reinforced steel tube under axial impact loads", International Journal of Impact Engineering, 122, 23-37, 2018

[23] Perillo, G., Vedivik, N.P. and Echtermeyer, A.T., "Damage development in stitch bonded GFRP composite plates under low velocity impact: Experimental and numerical results", Composite materials, 49(5), 601-615 2015. 